\title{
Risk factors for acquisition of colistin-resistant Klebsiella pneumoniae and expansion of a colistin- resistant ST307 epidemic clone in hospitals in Marseille, France, 2014 to 2017
}

Sophie Alexandra Baron ${ }^{1,2}$, Nadim Cassir ${ }^{1,2}$, Mouna Hamel ${ }^{1,2}$, Linda Hadjadj ${ }^{1,2}$, Nadia Saidani'2,3, Gregory Dubourg ${ }^{1,2}$, Jean-Marc Rolain 1,2

1. Aix Marseille Univ, IRD, APHM, MEPHI, Faculté de Médecine et de Pharmacie, Marseille, France

2. IHU Méditerranée Infection, Faculté de Médecine et de Pharmacie, Marseille, France

3. Aix Marseille Univ, IRD, AP-HM, SSA, VITROME, Marseille, France

Correspondence: Jean-Marc Rolain (jean-marc.rolain@univ-amu.fr)

Citation style for this article:

Baron Sophie Alexandra, Cassir Nadim, Hamel Mouna, Hadjadj Linda, Saidani Nadia, Dubourg Gregory, Rolain Jean-Marc. Risk factors for acquisition of colistinresistant Klebsiella pneumoniae and expansion of a colistin-resistant ST307 epidemic clone in hospitals in Marseille, France, 2014 to 2017. Euro Surveill. 2021;26(21): $\mathrm{pii}=2000022$. https://doi.org/10.2807/1560-7917.ES.2021.26.21.2000022

Background: France is a low prevalence country for colistin resistance. Molecular and epidemiological events contributing to the emergence of resistance to colistin, one of the 'last-resort' antibiotics to treat multidrug-resistant Gram-negative infections, are important to investigate. Aim: This retrospective (2014 to 2017) observational study aimed to identify risk factors associated with acquisition of colistinresistant Klebsiella pneumoniae (CRKP) in hospitals in Marseille, France, and to molecularly characterise clinical isolates. Methods: To identify risk factors for CRKP, a matched-case-control (1:2) study was performed in two groups of patients with CRKP or colistin-susceptible $K$. pneumoniae respectively. Whole-genome-sequences (WGS) of CRKP were compared with $6,412 \mathrm{~K}$. pneumoniae genomes available at the National Center for Biotechnology Information (NCBI). Results: Multivariate analysis identified male sex and contact with a patient carrying a CRKP as significant independent factors $(p<0.05)$ for CRKP acquisition, but not colistin administration. WGS of nine of 14 CRKP clinical isolates belonged to the same sequence type (ST)307. These isolates were from patients who had been hospitalised in the same wards, suggesting an outbreak. Comparison of the corresponding strains' WGS to $K$. pneumoniae genomes in $\mathrm{NCBI}$ revealed that in chromosomal genes likely playing a role in colistin resistance, a subset of five specific mutations were significantly associated with ST307 ( $p<0.001)$. Conclusion: A ST307 CRKP clone was identified in this study, with specific chromosomal mutations in genes potentially implicated in colistin resistance. $\mathrm{ST}_{307}$ might have a propensity to be or become resistant to colistin, however confirming this requires further investigations.

\section{Introduction}

Since the mid-2000s, carbapenem resistance has led to the revival of colistin, a polymyxin, as a last resort antibiotic [1] to treat Gram-negative bacterial infections [2], including carbapenemase-producing Klebsiella pneumoniae [2]. In this regard, the recent emergence of colistin resistance in humans and animals, particularly via the mobile colistin resistance gene $(\mathrm{mcr})$ is concerning [1]. For K. pneumoniae, a study between 2015 and 2017, concerning 18 European countries estimated an overall colistin resistance rate of $5.4 \%$ [3]. Other surveillance activities in Europe found resistance to polymyxins in this species varying from $2.6 \%$ in carbapenem-susceptible strains to $31.9 \%$ in carbapenemresistant ones [4].

In the literature, previous use of colistin has been reported as a risk factor for colistin resistance. Nevertheless, colistin-resistant bacteria have also been isolated from people who had not previously been treated with this drug [5] and, for K. pneumoniae, some investigations point to other risk factors in patients, such as previous carbapenemase-producing $K$. pneumoniae colonisation, corticosteroid administration and prior hospitalisations. The aforementioned risk factors were mainly assessed in Greece [6] and Italy [7], where the rate of colistin resistance reached $30 \%$, which is much higher than in France [8]. In our hospitals in Marseille, France, susceptibility to colistin is only tested in certain circumstances (see methods). As 
TABLE 1

Univariate analysis of characteristics potentially presenting a risk factor for colistin-resistant Klebsiella pneumoniae acquisition, Marseille, France, 2014-2017 ( $\mathrm{n}=66$ patients)

\begin{tabular}{|c|c|c|c|c|}
\hline \multicolumn{2}{|l|}{ Characteristics } & $\begin{array}{l}\text { CRKP } \\
n=22\end{array}$ & $\begin{array}{l}\text { CSKP } \\
n=44\end{array}$ & $\mathrm{p}$ value \\
\hline \multicolumn{2}{|c|}{ Age of patients in years; median (range) } & $69(53-76)$ & $66(53-75)$ & NS \\
\hline \multicolumn{2}{|c|}{ Number of patients of male sex } & 18 & 19 & 0.004 \\
\hline \multicolumn{2}{|c|}{ Length of hospital stay in days; mean (standard deviation) } & $60.1(56.6)$ & $44.7(40.3)$ & $<0.001$ \\
\hline \multicolumn{2}{|c|}{ Delay before first positive sample in days; mean (standard deviation) } & $21.7(22.1)$ & $13.2(17.2)$ & 0.151 \\
\hline \multicolumn{2}{|c|}{ Recent travel; number of patients (\%) } & $2(9)$ & $3(7)$ & NS \\
\hline \multirow{4}{*}{$\begin{array}{l}\text { Type of ward; } \\
\text { number of patients (\%) }\end{array}$} & Intensive care unit & $15(68)$ & $30(68)$ & NS \\
\hline & Surgery services & $3(14)$ & $6(14)$ & NS \\
\hline & Medical services & $3(14)$ & $6(14)$ & NS \\
\hline & Neonatal intensive care unit & $1(5)$ & $2(5)$ & NS \\
\hline \multirow{7}{*}{$\begin{array}{l}\text { Type of sample; } \\
\text { number of patients (\%) }\end{array}$} & Respiratory & $6(27)$ & $13(30)$ & 0.848 \\
\hline & Urine & $6(27)$ & $18(41)$ & 0.279 \\
\hline & Blood & $1(5)$ & $4(9)$ & 0.911 \\
\hline & Bone & $1(5)$ & $2(5)$ & NS \\
\hline & Liquid puncture & $2(9)$ & $4(9)$ & NS \\
\hline & Cutaneous swab & $2(9)$ & $6(14)$ & 0.923 \\
\hline & Rectal swab & $7(32)$ & $14(32)$ & NS \\
\hline \multirow{10}{*}{$\begin{array}{l}\text { Comorbidies; } \\
\text { number of patients (\%) }\end{array}$} & Solid organ cancer & $8(36)$ & $7(16)$ & 0.062 \\
\hline & Haematologic cancer & $1(5)$ & $3(7)$ & NS \\
\hline & Cerebrovascular disease & $\mathrm{o}(\mathrm{o})$ & $6(14)$ & 0.167 \\
\hline & Liver disease & $1(5)$ & $2(5)$ & NS \\
\hline & Chronic renal failure & $2(9)$ & $7(16)$ & 0.706 \\
\hline & Chronic pulmonary disease & $2(9)$ & $5(11)$ & NS \\
\hline & Diabetes mellitus & $5(23)$ & $13(30)$ & 0.770 \\
\hline & Cardiovascular disease & $7(32)$ & $12(27)$ & 0.701 \\
\hline & Solid organ transplantation & $\mathrm{o}(\mathrm{o})$ & $3(7)$ & 0.545 \\
\hline & Charlson score $\geq 3$ & $16(73)$ & $32(73)$ & NS \\
\hline \multirow{10}{*}{$\begin{array}{l}\text { History; } \\
\text { number of patients (\%) }\end{array}$} & Previous surgery & $10(45)$ & $15(34)$ & 0.370 \\
\hline & Mechanical ventilation & $14(64)$ & $20(45)$ & 0.164 \\
\hline & Central venous catheter & $16(73)$ & $32(73)$ & NS \\
\hline & Intravenous home therapy & $3(14)$ & $4(9)$ & 0.678 \\
\hline & Urinary catheter & $16(73)$ & $25(57)$ & 0.209 \\
\hline & Haemodialysis & $4(18)$ & $3(7)$ & 0.210 \\
\hline & Recent bacterial infection & $12(55)$ & $13(30)$ & 0.048 \\
\hline & Previous isolation of an ESBL-producing bacteria & $3(14)$ & $7(16)$ & NS \\
\hline & Previous isolation of a carbapenemase producing bacteria & $11(50)$ & $6(14)$ & 0.006 \\
\hline & Contact with patient carrying a CRKP & $14(64)$ & $12(27)$ & 0.004 \\
\hline \multirow{7}{*}{$\begin{array}{l}\text { Previous treatment; } \\
\text { number of patients (\%) }\end{array}$} & Amoxicillin + clavulanic acid & $4(18)$ & $9(20)$ & NS \\
\hline & Piperacillin + clavulanic acid & $9(41)$ & 17 (39) & 0.859 \\
\hline & Third generation cephalosporin & $13(59)$ & $15(34)$ & 0.053 \\
\hline & Carbapenems (imipenem, ertapenem or meropenem) & $9(41)$ & $7(16)$ & 0.025 \\
\hline & Fluoroquinolones & $11(50)$ & $21(48)$ & 0.025 \\
\hline & Aminoglycosides & $15(68)$ & $15(34)$ & 0.009 \\
\hline & Colistin & $3(14)$ & $2(5)$ & 0.323 \\
\hline
\end{tabular}

CRKP: colistin-resistant Klebsiella pneumoniae; CSKP: colistin-susceptible Klebsiella pneumoniae; ESBL: extended-spectrum beta-lactamase; NS: non-significant $p$ value. 
TABLE 2

Independent risk factors for acquisition of colistinresistant Klebsiella pneumoniae found through multivariate analysis, Marseille, France, 2014-2017 ( $\mathrm{n}=66$ patients)

\begin{tabular}{|l|l|l|}
\hline Risk factors & OR $(95 \% \mathrm{Cl})$ & $\mathrm{p}$ value \\
\hline Male sex & $2.5(1.5-12.5)$ & 0.023 \\
\hline Contact with patient carrying a CRKP & $3.7(1.8-17.0)$ & 0.014 \\
\hline
\end{tabular}

$\mathrm{Cl}$ : confidence interval; CRKP: colistin-resistant Klebsiella pneumoniae; OR: odds ratio.

a result, the prevalence of colistin resistance cannot be estimated. Moreover, the mechanisms of colistin resistance as well as the risk factors associated with the acquisition of colistin-resistant organisms have thus far not been assessed in our setting.

The $K$. pneumoniae sequence type (ST)307 was first reported in a clinical case in 2009 in Pakistan but subsequent findings indicated that this ST might have already emerged in the mid-1990s $[9,10]$. ST307 has been involved in local hospital outbreaks in Africa, the Americas, Asia and Europe [9]. It is frequently associated with multidrug resistance, especially with CTX-M-15 extended-spectrum beta-lactamase (ESBL) and carbapenemase ( $K$. pneumoniae carbapenemase; $\mathrm{KPC}$ ) and it has even replaced the multidrug-resistant international ST258 in some countries such as Canada and Italy [11]. In 2018, a whole genome sequencing (WGS) and epidemiologic analysis of KPC-Klebsiella pneumoniae isolates in France found the emergence of several high-risk clones in the hospital environment including ST307 [12]. Among studies focusing on ST307 multidrug resistance however, few have investigated colistin resistance $[10,13,14]$. On the other hand, of $K$. pneumoniae STs reported to be colistin-resistant, those belonging to clonal complexes (CC)258 (ST258, $\mathrm{ST}_{512}$ and $\mathrm{ST}_{11}$ ) and $\mathrm{CC}_{15}$ have most frequently been identified [10].

The aim of our work was to find colistin-resistant $K$. pneumoniae (CRKP) isolated from patients hospitalised in Marseille university hospitals between February 2014 and May 2017, to molecularly characterise the strains, and to determine risk factors associated with CRKP acquisition. Because strains of ST307 dominated among the CRKP identified, these were also the object of further focus and epidemiological investigations.

\section{Methods}

\section{Collection of colistin-resistant Klebsiella} pneumoniae strains and microbiological procedures

This retrospective study was conducted on patients who had been hospitalised in Marseille university hospitals, France, from February 2014 to August 2017. These four hospitals (Timone, Conception, North, and
Sainte-Marguerite hospitals) have a total of 3,700 beds.

Our laboratory carries out the microbiological analysis of specimens collected in the four hospitals. Colistin susceptibility testing is performed on $K$. pneumoniae isolates that are susceptible to less than three families of antibiotics included in our routine antibiotic testing panel, or upon specific request. We selected all $K$. pneumoniae strains (one per patient) for which a colistin minimum inhibitory concentration (MIC) was $>2 \mu \mathrm{g} / \mathrm{mL}$ (performed by E-test or microdilution method) according to the European Committee on Antimicrobial Susceptibility Testing (EUCAST) recommendations [15]. Antimicrobial susceptibility testing for other antibiotics was done according both the EUCAST and Comité de l'Antibiogramme - Société Française de Microbiologie (CA-SFM) recommendations using the disk diffusion method on MuellerHinton (MH) agar (BioMérieux, Marcy l'Etoile, France) enriched with commercial antibiotic disks, and/or E-test (Biomérieux) as previously described [16].

\section{Study of risk factors for colistin-}

\section{resistant Klebsiella pneumoniae acquisition}

Cases were defined as patients for whom a CRKP had been isolated at least once in one sample, thus including patients colonised and/or infected with CRKP. Patients' clinical histories were obtained from their electronic medical records. Each case was included in the study only once, i.e. at the time of the first CRKP isolation. In order to assess the risk factors for CRKP carriage, cases were compared to control patients colonised or infected with colistin-susceptible $K$. pneumoniae (CSKP) strains isolated during the same study period. In this case-control analysis, controls were matched to cases (2:1) by age, type of ward (medical, surgical or intensive care) and type of sample. Comorbidities/conditions analysed were as follows: organ transplant recipient, chronic renal disease, haematologic or solid organ cancer (excluded if remission > 5 years), chronic pulmonary disease, diabetes mellitus, splenectomy, cerebrovascular disease, liver cirrhosis and heart disease. Modified Charlson scores $\geq 3$ [17] were calculated and we considered several aspects of the patient's recent medical history such as: previous surgery ( 1 year prior to the current study), previous antibiotic therapy ( $\$ 3$ months prior), recent history of bacterial infection ( 1 month prior), previous isolation of an ESBL or a carbapenemase-producing bacteria, use of mechanical ventilation, haemodialysis, urinary catheter and central venous catheter. The final diagnosis (e.g. infection or colonisation) defined by the practitioner in his final report was also retrieved.

\section{Statistical analysis}

The selected variables were compared by the chisquared test, Fisher's exact test and the Student's t-test as appropriate. Univariate analysis was twosided and a significant $p$ value was chosen at $p<0.05$. Multivariate analysis was performed by conditional 


\section{FIGURE 1}

Hierarchical tree of antimicrobial susceptibility testing results of sequenced colistin-resistant Klebsiella pneumoniae isolates, Marseille, France, 2014-2017 ( $\mathrm{n}=14$ isolates)

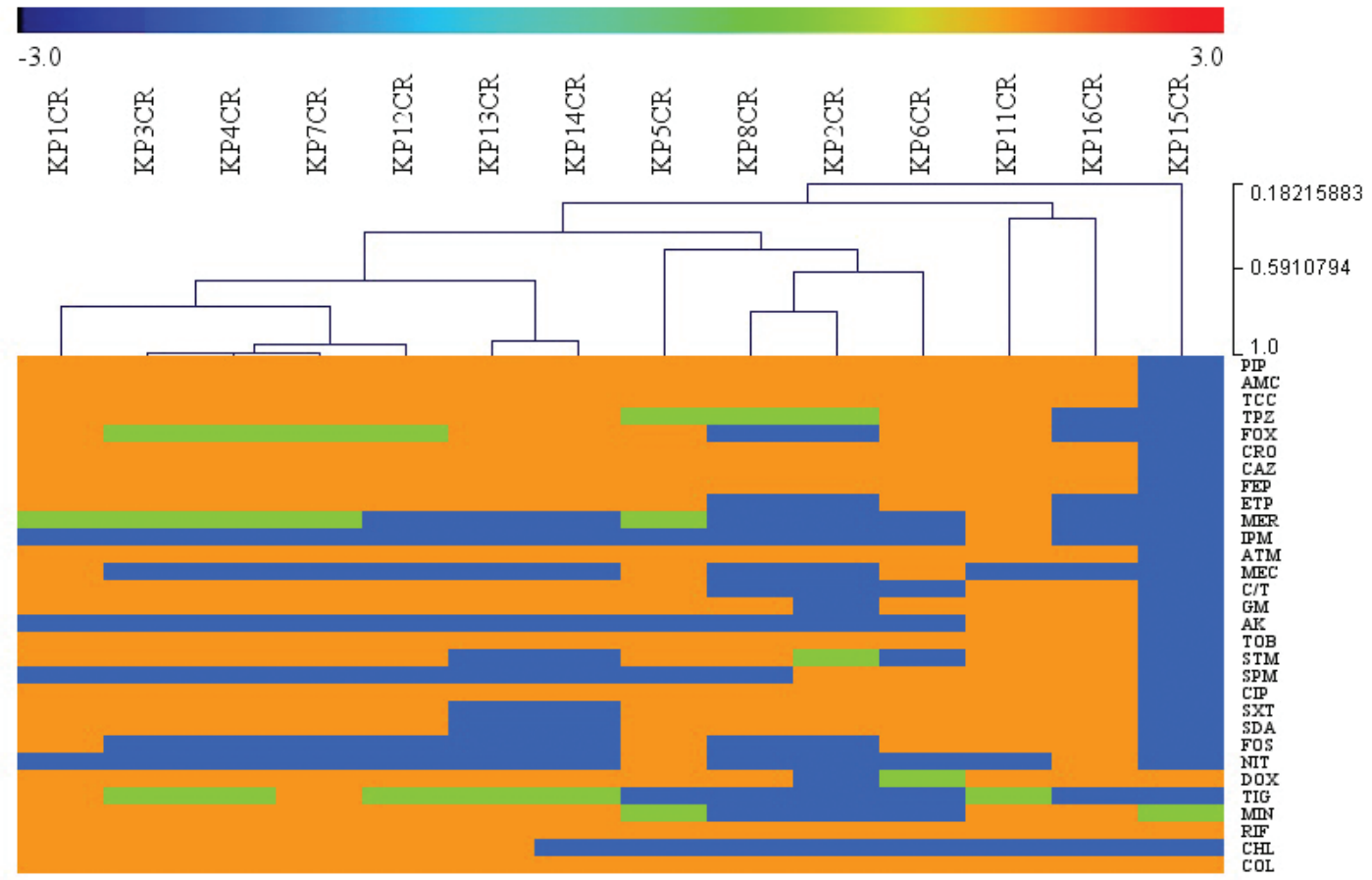

AK: amikacin; AMC: amoxicillin + clavulanic acid; ATM: aztreonam; CAZ: ceftazidime; CHL: chloramphenicol; CIP: ciprofloxacin; COL: colistin; CRO: ceftriaxone; C/T: ceftolozane + tazobactam; DOX: doxycyline; ETP: ertapenem; FEP: cefepime; FOS: fosfomycin; FOX: cefoxitin; GM: gentamicin; IPM: imipenem; MEC: mecillinam; MER: meropenem; MIN: minocycline; NIT: nitrofurantoin; PIP: piperacillin; RIF: rifampicin; SDA: sulfadiazine; SPM: spectinomycin; STM: streptomycin; SXT: trimethoprim + sulfamethoxazole; TCC: ticarcillin + clavulanic acid; TIG: tigecycline; TOB: tobramycin; TPZ: piperacillin + tazobactam.

The colistin resistant (CR) Klebsiella pneumoniae (KP) isolates/strains are listed above the diagram and in each column under them, the susceptibility profile to specific antibiotics, which are listed to the right, is depicted. Orange indicates that the strain is classified as resistant to an antibiotic, green that the strain is categorised as intermediate and blue that the strain is classified as susceptible.

logistic regression, and only the risk factors with a $\mathrm{p}$ value 0.05 in univariate analysis were used for the multivariate analysis. Statistical analysis was performed using the SPSS software (SPSS Inc, Chicago, Illinois, United States (US)).

\section{Genomic study}

Whole genome sequencing, bioinformatical analysis and clonal relationship

Genomic DNAs of $K$. pneumoniae strains were sequenced with the MiSeq Technology (Illumina, San Diego, US) with a $2 \times 250$ paired-end run strategy using the Nextera Mate Pair sample prep kit (Illumina). Genome assembly was done using the $A_{5}$-miseq software (http://sourceforge.net/projects/ngopt/) and annotated with Prokka (https://github.com/tseemann/ prokka). Plasmid replicons, virulence and resistance genes were sought with Abricate. Genome files were deposited in the National Center for Biotechnology Information (NCBI) database under the Bioproject number PRJNA520974 (Supplementary Table S1).

\section{Clonal relationship study}

Multilocus sequence typing (MLST) analysis was performed in silico to determine the ST of collected strains using the Bacterial Isolate Genome Sequence Database (BIGSdb) database (http://bigsdb.pasteur. $\mathrm{fr} /$ klebsiella/klebsiella.html). The wzi locus type was also identified with information from the BIGsdb database. The pangenome of $K$. pneumoniae isolates was determined using Roary [18]. We used in a first step the core gene alignment output file to construct the maximum likelihood phylogenetic tree using the RaXML software. In a second step, we performed a genome comparison based on single nucleotide polymorphism (SNP)s variants using the CSI Phylogeny (version 1.4) available on the Center for Genomic Epidemiology website (https://cge.cbs.dtu.dk/services/CSIPhylogeny). An average of 10 SNP (range: $0-30$ ) was chosen as cutoff to define isolates of a same clone as previously described [19].

In silico mutations analysis

Sequences of proteins involved in colistin resistance were compared with the colistin-susceptible $(\mathrm{MIC}=2 \mathrm{mg} / \mathrm{L}) \quad[20]$ reference strain $K$. pneumoniae MGH78578 (GenBank accession 
Phylogenetic tree based on core genome sequence alignment of colistin-resistant Klebsiella pneumoniae, Marseille, France, $2014-2017$ ( $n=14$ sequences)

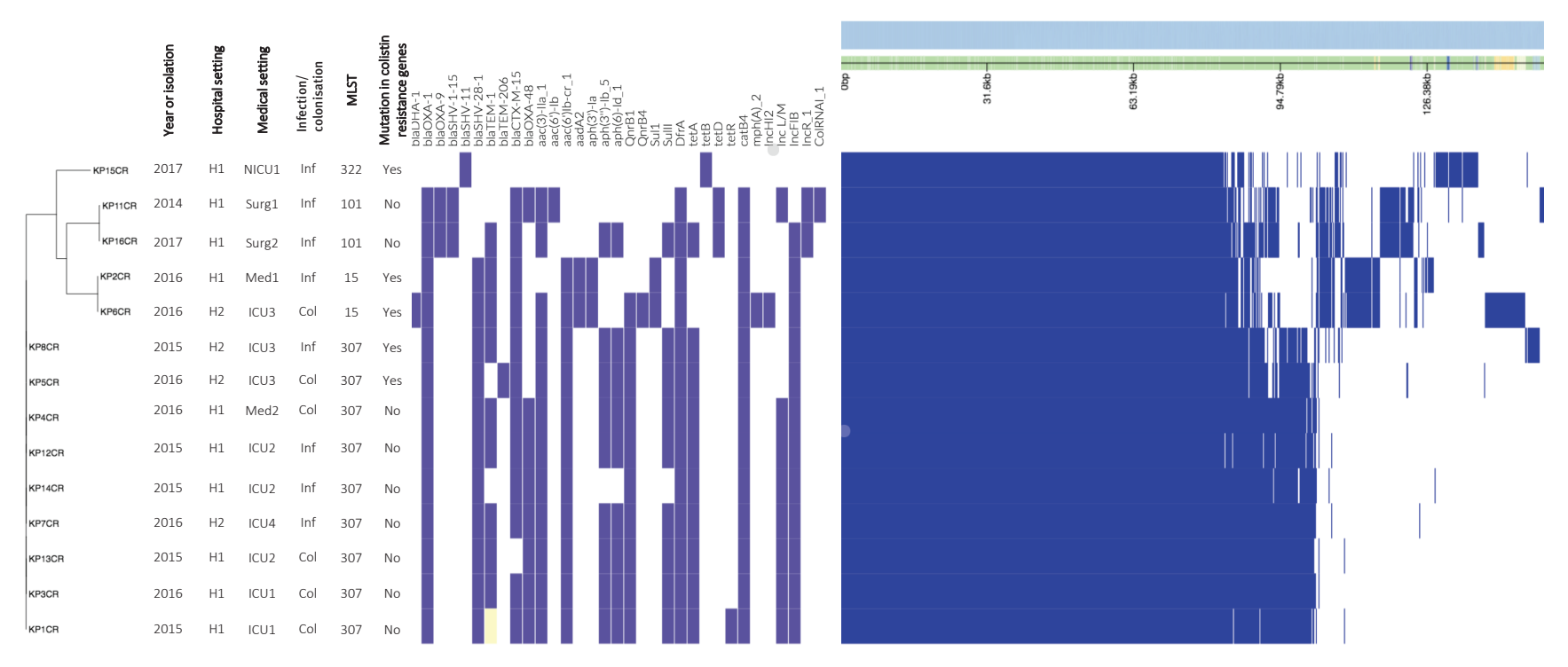

CR: colistin-resistant; ICU: intensive care unit; H: hospital; Inf: infection; KP: Klebsiella pneumoniae; Med: medical ward; MLST: multilocus sequence typing; Surg: surgery ward.

The following is indicated on the left side: year of isolation, the hospital, type of ward, stage of CRKP pathogenesis (colonisation/infection), MLST type and if a mutation was present in previously-studied colistin resistance genes. Other acquired resistant genes are indicated in purple if they are present in the genome. If a resistance gene is truncated, it is represented in yellow. On the right section of the Figure, the pangenomes of the K. pneumoniae strains are respectively represented, with on top a scale referring to the length of the pangenome. Each blue band represents a gene in a pangenome. Genes that are present in all pangenomes are toward the left of the section (core genome), whereas those toward the right, are not universally present in the pangenomes (accessory genome).

number: CPooo647). The deleterious effect of detected mutations on the function of proteins was investigated using Protein Variation Effect Analyzer (PROVEAN; $\quad$ http://provean.jcvi.org/index.php). Moreover, we investigated the frequency of the mutations found in PmrA/B, CrrA/B [20] and AcrR/S [21-23] in all ST307 among the 6,412 genomes of $K$. pneumoniae available on NCBI (last upgrade April 2019) by Basic Local Alignment Search Tool (BLAST) protein (blastp) using as threshold expect (E)-value $10 \mathrm{e}^{-5}$. Next we performed MLST using PubMLST typing schemes by local BLAST on all $K$. pneumoniae genomes available in $\mathrm{NCBI}$ to compare the frequency of different STs in this database.

\section{Ethical statement}

This study was qualified as an internal study not involving the human person, as it was conducted on the basis of data collected as part of the individual therapeutic or medical follow-up of patients, by the personnel providing this follow-up and for their exclusive use. It was approved by the sites' institutional review boards with a waiver of informed consent and registered under number RGPD/Ap-Hm 2020-02.

\begin{abstract}
Results
From February 2014 to August 2017, 22 patients carried a CRKP isolate among the 5,304 patients who had at least one $K$. pneumoniae isolated in our laboratory. During this period, 374 determinations of colistin MIC were performed. The median age of the 22 patients was 69years (range:53-76) and 18 were males (sex ratio $=4.5$ ). Four strains were isolated in 2014 , eight in 2015, seven in 2016 and three in 2017. Strains were recovered from five respiratory, six urine, and five stool samples as well as one blood culture, one bone biopsy, two liquid punctures and two cutaneous swabs. Colistin MIC varied from 4 to $>64 \mu \mathrm{g} / \mathrm{mL}$ (Supplementary Tables $\mathrm{S}_{2}$ and $\left.\mathrm{S}_{3}\right)$.
\end{abstract}

\section{Risk factors for colistin resistance acquisition}

The 22 CRKP cases were matched by age, type of ward and sample type with 44 controls carrying a CSKP isolated during the same study period. Univariate analysis of the two groups identified as significant risk factors male sex, length of hospital stay, recent bacterial infection, previous isolation of an ESBL or a carbapenemase producing bacteria and contact with cases carrying a CRKP (Table 1). Finally, previous antibiotic therapy, 
especially by fluoroquinolones, aminoglycosides or carbapenems was associated with an increased risk to acquire CRKP.

In the multivariate analysis, male sex $(p<0.05)$ and contact with a case carrying a CRKP $(p<0.01)$ remained the only independent factors for CRKP acquisition (Table 2).

In our study, only five patients had previously received colistin. Three cases with CRKP received at least intravenous colistin, while two patients with CSKP received only aerosolised colistin (Supplementary Table S4).

Twelve cases among the 22 in the CRKP group were infected vs 21 of 44 patients in the control group $(p=0.862)$. Mortality rate reached $18 \%(4 / 22)$ in the CRKP group vs $25 \%(11 / 44)$ in the CSKP group $(p=0.770)$. In the CRKP group, one death was attributable to $K$. pneumoniae infection.

\section{Clinical and biological features of cases with acquired colistin-resistant Klebsiella pneumoniae}

We compared cases with a CRKP infection to those who were just colonised by CRKP. The length of stay in hospital (37 vs 88 days; $p=0.025$ ) as well as the delay before the first positive CRKP sample (14 vs 31 days; $p=0.0385$ ) were significantly shorter in the infected group than in the colonised group. However, while the delay in the colonised group was based on 10 cases, only five of them had tested negative for CRKP at admission. The remainder had not been tested at that time, but were nevertheless used to estimate delay, under the assumption that testing was not performed, due to absence of risk factors requiring this. No other significant difference was observed between the two groups. No specific ST was associated with infection or colonisation.

Whole genome sequencing study and resistome As this work was a retrospective study and all laboratory strains are not permanently stored, only 14 of the 22 CRKP isolates from the microbiology laboratory were available for WGS analysis. Genomes sizes ranged from $5,518,326 \mathrm{bp}$ to $5,912,443 \mathrm{bp}$ with a percentage of GC bases ranging from 56.3 to $57.3 \%$. Genomes were assembled in 74 to 603 contigs with a 19 to 55 coverage. The main features of the different genomes are reported in Supplementary Tables S2 and S3.

Of 14 colistin-resistant $K$. pneumoniae strains, nine belonged to ST307, followed by two to ST15, two to ST101 and one to ST322. Antibiotic susceptibility testing was performed on the 14 sequenced strains (Supplementary Tables S2 and S3).

Resistance rate to each antibiotic is presented in Figure 1. The isolates were mostly susceptible to imipenem (13 isolates), nitrofurantoin (12 isolates), amikacin (12 isolates), minocycline (11 isolates), mecillinam (11 isolates), fosfomycin (nine isolates), chloramphenicol (eight isolates) and tigecycline (six isolates) (Figure 1). However, the combination of ceftolozane and tazobactam was active on only four isolates.

The nine ST307 CRKP carried bla ${ }_{O X A-1}, b l a_{S H V-28}$, $\operatorname{aac}(3)-\| a$, aac(6') $\mid b-c r$, qnrB1, tetA, and catB4 genes (Supplementary Tables $\mathrm{S}_{2}$ and $\mathrm{S}_{3}$ ). All but two (KP 5 CR and KP8CR) of these strains carried a bla $a_{O X A-48}$. The two ST15 CRKP (KP2CR and KP6CR on Figure 1) were positive for bla $a_{\text {OXA-1 }}, b l a_{\text {SHV-28 }}, b l a_{\text {TEM-1 }}$, bla $a_{\text {CTX-M-15 }}, a a c\left(6^{\prime}\right) l b-c r$, aadA2, aph(3')-la, sull, dfrA and catB4. One ST15 strain (KP6CR) was also carrying bla ${ }_{D H A-1}, a a c(3)-I l a, a n r B 1$, qnrB4, mph(A) genes. The two CRKP ST101 (KP11CR and KP16CR) had a bla $a_{\text {OXA-1 }}$, bla $a_{\text {OXA-9 }}$, bla $a_{\text {SHV }-15}$, bla $a_{\text {CTX- }}$ ${ }_{M-15}, a a c(3)-1 / a, d f r A$, tetD and catB4 genes. The strain KP 11 CR also carried aac (6') $l b$, bla ${ }_{\text {OXA-48 }}$ genes. The strain KP16CR was also positive for bla Finally, the ST322 strain (KP15CR), which was susceptible to all antibiotic but cyclins, was carrying a tet $B$ and a bla $a_{S H V-11}$ gene.

Spread of an epidemic ST307 colistinresistant Klebsiella pneumoniae in intensive care units

The pangenome of the 14 isolates recovered 7,897 genes, including 4,136 genes belonging to the core genome and 3,761 to the accessory genome (Figure 2). A phylogenetic tree based on the core genome showed that the nine ST307 strains were closely related (2-114 SNPs) and suggested that they could possibly belong to the same clone.

Interestingly, five of the nine $\mathrm{ST}_{30} 07$ cases $\left(\mathrm{KP}_{12} \mathrm{CR}\right.$, $\mathrm{KP} 13 \mathrm{CR}, \mathrm{KP} 14 \mathrm{CR}, \mathrm{KP} 1 \mathrm{CR}$ and $\mathrm{KP} 3 \mathrm{CR}$ ) met at different times between 2015 and 2016 in the same ward, i.e. intensive care unit (ICU)1 and ICU2 (Figure 3, light green and green) and became positive for CRKP in this ward. One additional case was present in the same ward (KP7CR) but never met the other CRKP positive cases. Another case ( $\left.\mathrm{KP}_{4} \mathrm{CR}\right)$, who had been previously hospitalised in a nearby town ICU and identified as a KPC carrier there, tested positive for KPCR on the first day of hospitalisation in Marseille. Interestingly, this person's respective isolate and the isolates of the six other cases only presented between 2 and 13 SNPs (average 7 SNPs), confirming that all seven were from the same clone. However, two other cases (KP8CR and $\mathrm{KP}_{5} \mathrm{CR}$ ) who were hospitalised in a common ward $\left(\mathrm{ICU}_{3}\right.$, red), never met and genomes recovered from their isolates had between 91 and 114 different SNPS (average 102 SNPs), suggesting that they were affected by different strains. Moreover, these two strains were negative for the $b a_{O X_{A 4} 8}$ gene that was present in the other ST307 strains.

\section{Possible genetic basis of colistin resistance in Klebsiella pneumoniae strains}

All strains were negative for the $m c r-1$ to $m c r-8$ gene variants. Mutations for the most common genes involved in colistin resistance were analysed (Table 3). All 14 strains analysed showed mutations in at least 
Time of hospitalisations in different wards of cases of Klebsiella pneumoniae ST307 resistant to colistin and time of the respective bacterial isolation, Marseille, France, 2015-2016 ( $\mathrm{n}=9$ cases)

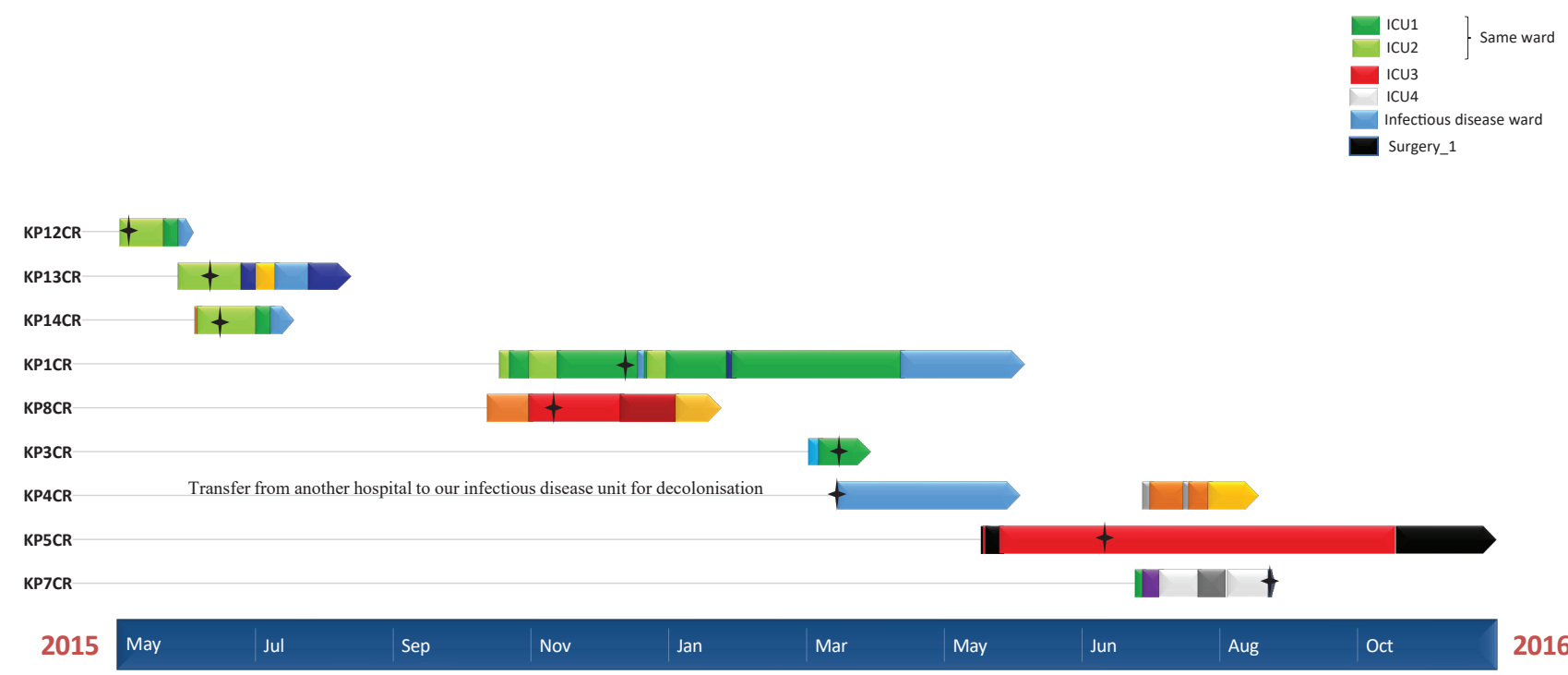

ST: sequence type; KP: Klebsiella pneumoniae; CR: colistin resistant.

Each colour represents a different ward (only the wards of interest are described in the legend). The units in light green and green correspond to two units of the same ward; patients are often transferred between these two units according to the severity of their clinical conditions. The star indicates the first detection of a CRKP.

one colistin-resistance gene compared with the reference strain $\mathrm{MGH} 78578$. In nine of these, no mutation predicted or known to be responsible for colistin resistance was detected in any of the genes subjected to PROVEAN analysis.

Five isolates (35.7\%) had mutations in the pmrA, p hoP, phoQ or mgrB genes and these mutations were predicted to modify protein function according to PROVEAN analysis (Table 3 ). We also noticed five mutations (PmrA A41T, PmrB L213M and T246A, CrrB C68S, AcrS S76R) that were present in all ST307 isolates. These were predicted by PROVEAN to not alter protein function independently. To explore if they might have, together, a synergistic role in colistin resistance and/or if they might be specific for ST307, we investigated the frequency of these mutations in 6,412 genomes of $K$. pneumoniae available on NCBI. Interestingly, simultaneous presence of the five mutations was found in 195 of 6,412 genomes analysed, including all 192 ST307 present in GenBank, one ST2739 (that belongs to C(307), one ST2975 and one genome with an unknown ST (Supplementary Figure S1). The frequency of the five mutations was strongly associated with the ST307 (192/195 vs 3/6,217; p<0.001). Unfortunately, colistin susceptibility data were mostly lacking from the metadata of these genomes, preventing any insight into any potential association with colistin resistance.

Taken one by one, the frequency of each mutation in the 6,412 genomes was as follows: $3.2 \%(n=204)$ of
A41T in PmrA, 69.8\% $(n=4,474)$ of $\mathrm{C} 68 \mathrm{~S}$ in CrrB and $3.3 \%(n=209)$ of S76R in AcrS (Supplementary Table $\mathrm{S}_{5}$ ). A total of 288 genomes $(4.5 \%)$ had mutations leading to presence of both $\mathrm{L} 213 \mathrm{M}$ and T246A in the PmrB protein.

In terms of the occurrence of each ST or CC among the 6,412 genomes in GenBank, CC258 was most prevalent $(n=1,411 ; 22 \%)$, followed by ST11 $(n=715 ; 11 \%)$, ST15 $(n=336 ; 5 \%)$ ST101 $(n=206 ; 3 \%)$ and ST307 ( $n=192$; 3\%) (Supplementary Figure S1).

\section{Discussion}

In this study, we retrospectively found 22 CRKP cases in four Marseille university hospitals. Univariate analysis identified several characteristics associated with carriage or infection with CRKP including male sex, length of hospital stay, recent bacterial infection, contact with a patient positive for CRKP and previous antibiotic therapy with fluoroquinolones, aminoglycosides and carbapenems. Furthermore, previous isolation of a carbapenemase-producing bacteria in patients was also determined as a risk factor, suggesting that controlling carbapenem resistance may possibly help mitigate emergence of CRKP in this setting.

In multivariate analysis, however, only male sex and contact with another patient carrying a CRKP remained as independent risk factors for CRKP acquisition. Therefore management options for patients carrying or infected by a CRKP strain could include isolation 
TABLE 3

Summary of mutations found in the 14 strains of Klebsiella pneumoniae analysed, Marseille, France, $2014-2017$ ( $\mathrm{n}=14$ isolates)

\begin{tabular}{|c|c|c|c|c|c|c|c|c|c|c|c|c|c|}
\hline Strain & ST & $w z i$ & $\begin{array}{l}\text { Colistin MIC } \\
(\mu \mathrm{g} / \mathrm{mL})\end{array}$ & PmrA & PmrB & PhoP & PhoQ & MgrB & CrrB & CrrA & AcrR & AcrS & $\mathrm{mcr}$ \\
\hline $\mathrm{KP} 1 \mathrm{CR}$ & 307 & $\mathrm{NF}^{\mathrm{a}}$ & 16 & A41T [13] & $\begin{array}{c}\text { L213M [13]; } \\
\text { T246A }[26,37]\end{array}$ & $\mathrm{No}^{\mathrm{b}}$ & $\mathrm{No}^{\mathrm{b}}$ & $\mathrm{No}^{\mathrm{b}}$ & C68S & $\mathrm{No}^{\mathrm{b}}$ & $\mathrm{No}^{\mathrm{b}}$ & S76R & $\mathrm{No}^{\mathrm{b}}$ \\
\hline $\mathrm{KP} \mathrm{P}_{2} \mathrm{CR}$ & 15 & $\mathrm{NF}^{\mathrm{a}}$ & 16 & $\mathrm{G}_{53} \mathrm{~V}^{\mathrm{c}}[26]$ & $\mathrm{No}^{\mathrm{b}}$ & $\mathrm{No}^{\mathrm{b}}$ & $\mathrm{No}^{\mathrm{b}}$ & $\mathrm{No}^{\mathrm{b}}$ & $\mathrm{NF}^{\mathrm{a}}$ & $\mathrm{NF}^{\mathrm{a}}$ & $\mathrm{No}^{\mathrm{b}}$ & Nob & $\mathrm{No}^{\mathrm{b}}$ \\
\hline $\mathrm{KP} \mathrm{P}_{3} \mathrm{CR}$ & 307 & $\mathrm{NF}^{\mathrm{a}}$ & 16 & $\mathrm{~A} 41 \mathrm{~T}$ & L213M; T246A & $\mathrm{No}^{\mathrm{b}}$ & $\mathrm{No}^{\mathrm{b}}$ & $\mathrm{No}^{\mathrm{b}}$ & $\mathrm{C} 68 \mathrm{~S}$ & $\mathrm{No}^{\mathrm{b}}$ & $\mathrm{No}^{\mathrm{b}}$ & S76R & $\mathrm{No}^{\mathrm{b}}$ \\
\hline $\mathrm{KP} 4 \mathrm{CR}$ & 307 & 173 & 8 & A41T & L213M; T246A & $\mathrm{No}^{\mathrm{b}}$ & $\mathrm{No}^{\mathrm{b}}$ & $\mathrm{No}^{\mathrm{b}}$ & C68S & $\mathrm{No}^{\mathrm{b}}$ & $\mathrm{No}^{\mathrm{b}}$ & S76R & $\mathrm{No}^{\mathrm{b}}$ \\
\hline $\mathrm{KP}_{5} \mathrm{CR}$ & 307 & 173 & 16 & $\mathrm{~A}_{41 \mathrm{~T}}$ & L213M; T246A & $\mathrm{L} 12 \mathrm{Q}$ & $\mathrm{No}^{\mathrm{b}}$ & $\mathrm{No}^{\mathrm{b}}$ & $\mathrm{C} 68 \mathrm{~S}$ & $\mathrm{No}^{\mathrm{b}}$ & $\mathrm{No}^{\mathrm{b}}$ & S76R & $\mathrm{No}^{\mathrm{b}}$ \\
\hline KP6CR & 15 & $\mathrm{NF}^{\mathrm{a}}$ & 16 & $\mathrm{No}^{\mathrm{b}}$ & $\mathrm{No}^{\mathrm{b}}$ & $\mathrm{No}^{\mathrm{b}}$ & $\mathrm{L} 87 \mathrm{P}$ & $\mathrm{No}^{\mathrm{b}}$ & $\mathrm{NF}^{\mathrm{a}}$ & $\mathrm{NF}^{\mathrm{a}}$ & $\mathrm{No}^{\mathrm{b}}$ & $\mathrm{No}^{\mathrm{b}}$ & $\mathrm{No}^{\mathrm{b}}$ \\
\hline $\mathrm{KP} 7 \mathrm{CR}$ & 307 & 173 & 16 & $\mathrm{~A} 41 \mathrm{~T}$ & L213M; T246A & $\mathrm{No}^{\mathrm{b}}$ & $\mathrm{No}^{\mathrm{b}}$ & $\mathrm{No}^{\mathrm{b}}$ & $\mathrm{C} 68 \mathrm{~S}$ & $\mathrm{No}^{\mathrm{b}}$ & $\mathrm{No}^{\mathrm{b}}$ & S76R & $\mathrm{No}^{\mathrm{b}}$ \\
\hline KP8CR & 307 & 173 & 32 & $\mathrm{~A} 41 \mathrm{~T}$ & L213M; T246A & $\mathrm{No}^{\mathrm{b}}$ & $\mathrm{No}^{\mathrm{b}}$ & $\begin{array}{c}\text { Stop } \\
(13 A A)^{d}\end{array}$ & $\mathrm{C} 68 \mathrm{~S}$ & $\mathrm{No}^{\mathrm{b}}$ & $\mathrm{No}^{\mathrm{b}}$ & S76R & $\mathrm{No}^{\mathrm{b}}$ \\
\hline KP11CR & 101 & 137 & 16 & $\mathrm{~A} 217 \mathrm{~V}$ & T246A & $\mathrm{No}^{\mathrm{b}}$ & $\mathrm{No}^{\mathrm{b}}$ & $\mathrm{No}^{\mathrm{b}}$ & $\mathrm{NF}^{\mathrm{a}}$ & $\mathrm{NF}$ & $\mathrm{No}^{\mathrm{b}}$ & $\mathrm{H} 79 \mathrm{Q}$ & $\mathrm{No}^{\mathrm{b}}$ \\
\hline $\mathrm{KP} 12 \mathrm{CR}$ & 307 & 173 & 8 & $\mathrm{~A} 41 \mathrm{~T}$ & L213M; T246A & $\mathrm{No}^{\mathrm{b}}$ & $\mathrm{No}^{\mathrm{b}}$ & $\mathrm{No}^{\mathrm{b}}$ & $\mathrm{C} 68 \mathrm{~S}$ & $\mathrm{No}^{\mathrm{b}}$ & $\mathrm{No}^{\mathrm{b}}$ & S76R & $\mathrm{No}^{\mathrm{b}}$ \\
\hline $\mathrm{KP} 13 \mathrm{CR}$ & 307 & 173 & 16 & $\mathrm{~A} 41 \mathrm{~T}$ & L213M; T246A & $\mathrm{No}^{\mathrm{b}}$ & $\mathrm{No}^{\mathrm{b}}$ & $\mathrm{No}^{\mathrm{b}}$ & C68S & $\mathrm{No}^{\mathrm{b}}$ & $\mathrm{No}^{\mathrm{b}}$ & S76R & $\mathrm{No}^{\mathrm{b}}$ \\
\hline $\mathrm{KP} 14 \mathrm{CR}$ & 307 & 173 & 4 & $\mathrm{~A}_{41 \mathrm{~T}}$ & L213M; T246A & $\mathrm{No}^{\mathrm{b}}$ & $\mathrm{No}^{\mathrm{b}}$ & $\mathrm{No}^{\mathrm{b}}$ & $\mathrm{C} 68 \mathrm{~S}$ & $\mathrm{No}^{\mathrm{b}}$ & $\mathrm{No}^{\mathrm{b}}$ & S76R & $\mathrm{No}^{\mathrm{b}}$ \\
\hline $\mathrm{KP}_{15} \mathrm{CR}$ & 322 & 50 & $>64$ & S64T [26] & $\mathrm{No}^{\mathrm{b}}$ & $\mathrm{No}^{\mathrm{b}}$ & $\mathrm{No}^{\mathrm{b}}$ & $\begin{array}{c}\text { IS10 } \\
\text { in bp } \\
76[38]\end{array}$ & C68S & $\mathrm{No}^{\mathrm{b}}$ & $\mathrm{No}^{\mathrm{b}}$ & P211S & $\mathrm{No}^{\mathrm{b}}$ \\
\hline KP16CR & 101 & 137 & 4 & $\mathrm{~A} 217 \mathrm{~V}[13]$ & T246A & $\mathrm{No}^{\mathrm{b}}$ & $\mathrm{N} 135 \mathrm{~K}$ & $\mathrm{No}^{\mathrm{b}}$ & $\mathrm{NF}^{\mathrm{a}}$ & $N F^{a}$ & $\mathrm{No}^{\mathrm{b}}$ & $\mathrm{H} 79 \mathrm{Q}$ & $\mathrm{No}^{\mathrm{b}}$ \\
\hline
\end{tabular}

AA: amino acid; CR: colistin resistant; IS: Insertion Sequence; KP: Klebsiella pneumoniae; MIC: minimum inhibitory concentration; NF: not founda; PROVEAN: Protein Variation Effect Analyzer; ST: sequence type.

a The gene was not found.

${ }^{b}$ No mutation was found in the gene compared with the reference strain $\mathrm{MGH}_{7} 8578$.

${ }^{c} \mathrm{~A}$ mutation at this position was previously reported to alter protein function, but the substitution was $\mathrm{G} 53 \mathrm{C}$.

d Stop codon at the codon for the 13 th AA, leading to a truncated protein.

Mutations in bold were predicted to modify protein function by PROVEAN analysis. Studies that have previously reported some of the mutations listed in the Table are respectively cited next to the mutation.

by trained staff, decolonisation and/or possibly faecal microbiota transplantation. Interestingly, in terms of patient care, a large proportion of the strains from the CRKP cases analysed here, were susceptible to common antibiotics that have been used in the past (e.g. mecillinam, nitrofurantoin, fosfomycin) [2]. Such antibiotics could represent valuable treatment alternatives in cases of CRKP infection.

The results of our case-control analysis confirmed those of other studies (Supplementary Table S6), though we did not identify colistin administration as a risk factor for CRKP acquisition. It should be nevertheless noted, that in our study, only five patients received colistin (two in the CSKP group vs three in the CRKP group), resulting in relatively weak statistical power. Comparison between groups of cases and controls showed no difference in mortality. This finding differs from a few other previous investigations, which uncovered an excess mortality rate in cases of CRKP infections [7,24]; however, these studies focused on colistin-resistant carbapenemase-producing $K$. pneumoniae strains. Moreover, in our study, patients in both groups had various comorbidities that could have been confounding.

The $K$. pneumoniae isolates retrospectively considered for this study were selected based on colistin resistance only, without prior knowledge of their type. Of the 14 CRKP cases' isolates, where sequencing was possible, nine were $\mathrm{ST} 307$, with seven of these belonging to the same clone. The two CRKP cases respectively bearing separate ST307 clones, never met. Among the seven cases affected by the same ST307 clone, five were hospitalised in the same ICUs at different - but sometimes overlapping - times between 2015 and 2016. As has already been demonstrated in colistinresistance events [25], this might suggest cross-transmission. In this event, cross-transmission could have occurred in the ICU(s) via healthcare workers and/or environmental contamination. For the two remaining single-clone-affected CRKP cases, one had stayed in the same ICU as the other five, but at a completely different time, never meeting them. The other was never hospitalised in ICU, but was admitted to an infectious disease ward after transfer from another hospital near Marseille. This might indicate that the ST307 strain could present as an epidemic colistin-resistant clone, which has spread in our area, especially in the hospital environment $[9,10]$. In this regard, a national survey in France in 2014 detected three isolates with ST307 CRKP originating from the region Provence-Alpes-Côte d'Azur, to which Marseille belongs [8]. A French study in 2018 showed ST307 emerging in other areas of France, reinforcing its relevance [12]. 
Resistance to colistin in $K$. pneumoniae has previously been linked to several mechanisms including capsule overexpression, modifications of lipid A by addition of aminoarabinose or phosphoethanolamine due to mutations of target chromosomal genes or acquisition of plasmid-borne colistin resistance mor genes [1]. All strains in our study were negative for $\mathrm{mcr}$ genes. However, among the 14 isolates analysed by WGS, substitutions, deletions and insertions in genes known to play a role colistin resistance, and likely to result in functional change at the protein level according to PROVEAN, were identified in five strains (two ST15, two ST307 and one ST322). In two of these (ST322 and ST15), the $\operatorname{mgr} B$ gene was inactivated by an insertion sequence ( $\mathrm{S}_{10}$ ) and by a mutation leading to a stop codon respectively, while in another (ST307), a substitution occurred at position 53 of PmrA (Table 3 ). Remarkably, mutations in MgrB have previously been reported to lead to a high MIC of colistin $[1,26]$ as well as a mutation at position 53 in PmrA [26]. The two other mutations (PhoP L12Q and PhoQ P87L), which were respectively found in one $\mathrm{ST}_{307}$ and one $\mathrm{ST}_{15}$ strain, have never been described before and in vitro tests should be performed to confirm their role in colistin resistance.

Unfortunately, for the moment, we were not able to clearly decipher the molecular mechanism of colistin resistance for the remaining nine strains, two of which were ST101 and seven ST307. Of the latter, it can nevertheless be noted that five (i.e. $\mathrm{KP}_{4} \mathrm{CR}, \mathrm{KP} 7 \mathrm{CR}, \mathrm{KP}_{12} \mathrm{CR}$, $\mathrm{KP} 13 \mathrm{CR}$ and $\mathrm{KP} 14 \mathrm{CR}$ ) had identical mutations compared to the reference strain $M G H 78578$, but exhibited various colistin MICs. A different level of gene expression might explain these MIC differences, and this could be explored by transcriptomic analysis in further work. The mutations present in these five $\mathrm{ST} 307$ isolates (A41T in PmrA, L213M and T246A in PmrB, S76R in AcrS and $\mathrm{C} 68 \mathrm{~S}$ in $\mathrm{CrrB}$ ) were also present in all nine ST307 sequences recovered from our CRKP cases. Subsequent finding that all 192 ST307 bacterial genomes in NCBI, as at April 2019, also shared these mutations was surprising. Unfortunately, colistin susceptibility data are mostly lacking from the metadata of these genomes, preventing insight into the relation of these mutations with colistin resistance. Further work is needed to study any possible association (Supplementary Table S7).

Interestingly, seven of the nine ST307 from our CRKP cases had the wzi-173 allele which seems to be associated with this ST [11] and which confers an additional capsular locus that may distinguish this particular phenotype [1].

During the $29^{\text {th }}$ European Congress of Clinical Microbiology and Infectious Diseases (ECCMID) in Amsterdam, the Netherlands (13-16 April 2019), ST307 was reported as an emerging clone in several countries including Italy [27,28], Russia [29], Qatar [30], France [31], South Africa [32], Spain [33] and the Netherlands
[34]. From these reports, colistin resistance was found in 15 of 30 ST307 isolates detected in the Italian study and 24 of 35 isolates in the Russian one. Moreover, among a collection of 27 colistin-resistant K. pneumoniae from Kentucky, US [35], three were ST307. This raises the question as to whether our ST307 isolates from Marseille, France, are intrinsically resistant to colistin or if there is a propensity of $\mathrm{ST}_{30} 07$ clone to be or to become resistant to colistin everywhere in the world.

We previously reported a case of an osteitis caused by a ST307 CSKP [36] treated with colistin, highlighting that this lineage was already present in our hospital centre in 2015. This isolate (Genbank accession number: NJGM01) had the same mutations in PmrA (A41T), PmrB (L213M and T216A) and AcrR (S76R) but the CrrAB proteins were absent. A SNP analysis between this colistin susceptible isolate and the colistin-resistant ST307 isolates from the current study (used as references) found between 995 and 1,546 SNPs (mean=1,333) on an average of 5,540,016 bp analysed. The ST307 CRKP could have evolved from a CSKP clone in our setting. An exhaustive analysis of a larger sample collected from different sources would confirm this hypothesis. This study has some limitations. The first is related to the retrospective nature of the study, which limited access to some clinical and laboratory data, such as the results from screening the patient for multidrugresistant bacteria upon admission to hospital. The restrospective nature also impacted the availability of strains for sequencing from patients included in the study. Moreover as our initial aim was to molecularly characterise CRKP, no strains from patients in the CSKP control group for the case-control analysis were sequenced. The ST characterisation in this group might have provided more information on any relation between ST307 and the 'colistin-resistant' phenotype. The previous description of a carbapenemase-producing but colistin-susceptible $K$. pneumoniae ST307 strain suggests, however, that different clones of ST307 have diffused in our hospital, at least one susceptible and some resistant to colistin. Finally, as seven patients carried the same clone, this could have influenced the results of risk-factor analysis if there had been a clonehost relationship, such as a propensity of the clone to infect the same type of tissue or patient, however we did not observe this in our study.

In conclusion, this study concurs with previous ones, on risk factors for acquisition of CRKP and documents a CRKP ST307 epidemic clone in hospitals in Marseille, France, which is a country with an overall low prevalence of colistin resistance. Further work is warranted to understand why some clones have an ability to become increasingly resistant to antibiotics, by plasmid or chromosomal mechanism, as appears to be the case for CC258 [10] and ST307. 


\section{Acknowledgements}

We want to thank the technicians from our genomic platform for technical assistance and CookieTrad for English corrections.

\section{Conflict of interest}

None declared.

\section{Authors' contributions}

SB, GD, NC and JMR designed the study and drafted and revised the manuscript.

SB, NS and NC performed medical examinations and/or examined medical records.

SB and LH performed microbiology analyses.

SB and MH performed genomic analyses.

All authors have read and approved the final manuscript.

\section{References}

1. Baron S, Hadjadj L, Rolain J-M, Olaitan AO. Molecular mechanisms of polymyxin resistance: knowns and unknowns. Int J Antimicrob Agents. 2016;48(6):583-91. https://doi. org/10.1016/j.ijantimicag.2016.06.023 PMID: 27524102

2. Cassir N, Rolain J-M, Brouqui P. A new strategy to fight antimicrobial resistance: the revival of old antibiotics. Front Microbiol. 2014;5:551. https://doi.org/10.3389/ fmicb.2014.00551 PMID: 25368610

3. Stone GG, Seifert H, Nord CE. In vitro activity of ceftazidimeavibactam against Gram-negative isolates collected in 18 European countries, 2015-2017. Int J Antimicrob Agents. 2020;56(3):106045. https://doi.org/10.1016/j. ijantimicag.2020.106045 PMID: 32522673

4. European Centre for Disease Prevention and Control (ECDC). Antimicrobial resistance surveillance in Europe 2015. Annual Report of the European Antimicrobial Resistance Surveillance Network (EARS-Net). Stockholm: ECDC; 2017 . Available from: https://www.ecdc.europa.eu/sites/portal/files/media/ en/publications/Publications/antimicrobial-resistanceeurope-2015.pdf

5. Olaitan AO, Morand S, Rolain J-M. Emergence of colistinresistant bacteria in humans without colistin usage: a new worry and cause for vigilance. Int J Antimicrob Agents. 2016;47(1):1-3. https://doi.org/10.1016/j. ijantimicag.2015.11.009 PMID: 26712133

6. Zarkotou O, Pournaras S, Voulgari E, Chrysos G, Prekates A, Voutsinas D, et al. Risk factors and outcomes associated with acquisition of colistin-resistant KPC-producing Klebsiella pneumoniae: a matched case-control study. J Clin Microbiol. 2010;48(6):2271-4. https://doi.org/10.1128/JCM.02301-09 PMID: 20375234

7. Giacobbe DR, Del Bono V, Trecarichi EM, De Rosa FG, Giannella M, Bassetti M, et al. Risk factors for bloodstream infections due to colistin-resistant KPC-producing Klebsiella pneumoniae: results from a multicenter case-control-control study. Clin Microbiol Infect. 2015;21(12):1106.e1-8. https://doi. org/10.1016/j.cmi.2015.08.001 PMID: 26278669

8. Jayol A, Poirel L, Dortet L, Nordmann P. National survey of colistin resistance among carbapenemase-producing Enterobacteriaceae and outbreak caused by colistin-resistant OXA-48-producing Klebsiella pneumoniae, France, 2014. Euro Surveill. 2016;21(37):30339. https://doi.org/10.2807/15607917.ES.2016.21.37.30339 PMID: 27685838

9. Wyres KL, Hawkey J, Hetland MAK, Fostervold A, Wick RR, Judd LM, et al. Emergence and rapid global dissemination of CTX-M15-associated Klebsiella pneumoniae strain ST307. J Antimicrob Chemother. 2019;74(3):577-81. https://doi.org/10.1093/jac/ dky492 PMID: 30517666

10. David S, Reuter S, Harris SR, Glasner C, Feltwell T, Argimon S, et al. Epidemic of carbapenem-resistant Klebsiella pneumoniae in Europe is driven by nosocomial spread. Nat Microbiol. 2019;4(11):1919-29. https://doi.org/10.1038/s41564-019-04928 PMID: 31358985
11. Peirano G, Chen L, Kreiswirth BN, Pitout JDD. Emerging Antimicrobial-Resistant High-Risk Klebsiella pneumoniae clones ST307 and ST147. Antimicrob Agents Chemother. 2020;64(10):e01148--20. https://doi.org/10.1128/AAC.0114820 PMID: 32747358

12. Bonnin RA, Jousset $A B$, Chiarelli A, Emeraud C, Glaser P, Naas $\mathrm{T}$, et al. Emergence of New Non-Clonal Group 258 High-Risk Clones among Klebsiella pneumoniae CarbapenemaseProducing K. pneumoniae Isolates, France. Emerg Infect Dis. 2020;26(6):1212-20. https://doi.org/10.3201/eid26o6.191517 PMID: 32441629

13. Novović K, Trudić A, Brkić S, Vasiljević Z, Kojić M, Medić D, et al. Molecular Epidemiology of Colistin-Resistant, Carbapenemase-Producing Klebsiella pneumoniae in Serbia from 2013 to 2016. Antimicrob Agents Chemother. 2017;61(5):e02550-16. https://doi.org/10.1128/AAC.02550-16 PMID: 28242665

14. Castanheira M, Farrell SE, Wanger A, Rolston KV, Jones RN, Mendes RE. Rapid expansion of KPC-2-producing Klebsiella pneumoniae isolates in two Texas hospitals due to clonal spread of ST258 and ST307 lineages. Microb Drug Resist. 2013;19(4):295-7. https://doi.org/10.1089/mdr.2012.0238 PMID: 23530541

15. The European Committee on Antimicrobial Susceptibility Testing (EUCAST). Breakpoint tables for interpretation of MICs and zone diameters. Version 6.0. Växjö: EUCAST; 2016.

16. Le Page S, Dubourg G, Baron SA, Rolain J-M, Raoult D. No global increase in resistance to antibiotics: a snapshot of resistance from 2001 to 2016 in Marseille, France. Eur ] Clin Microbiol Infect Dis. 2019;38(2):395-407. https://doi. org/10.1007/s10096-018-3439-8 PMID: 30515637

17. Quan H, Li B, Couris CM, Fushimi K, Graham P, Hider P, et al. Updating and validating the Charlson comorbidity index and score for risk adjustment in hospital discharge abstracts using data from 6 countries. Am J Epidemiol. 2011;173(6):676-82. https://doi.org/10.1093/aje/kwq433 PMID: 21330339

18. Page AJ, Cummins CA, Hunt M, Wong VK, Reuter S, Holden MTG, et al. Roary: rapid large-scale prokaryote pan genome analysis. Bioinformatics. 2015;31(22):3691-3. https://doi. org/10.1093/bioinformatics/btv421 PMID: 26198102

19. Pérez-Vázquez M, Oteo J, García-Cobos S, Aracil B, Harris $\mathrm{SR}$, Ortega $\mathrm{A}$, et al. Phylogeny, resistome and mobile genetic elements of emergent OXA-48 and OXA-245 Klebsiella pneumoniae clones circulating in Spain. J Antimicrob Chemother. 2016;71(4):887-96. https://doi.org/10.1093/jac/ dkv458 PMID: 26769896

20. Wand ME, Bock LJ, Sutton JM. Retention of virulence following colistin adaptation in Klebsiella pneumoniae is straindependent rather than associated with specific mutations. Med Microbiol. 2017;66(7):959-64. https://doi.org/10.1099/ jmm.0.000530 PMID: 28741998

21. Hirakawa H, Takumi-Kobayashi A, Theisen U, Hirata T, Nishino $\mathrm{K}$, Yamaguchi A. AcrS/EnvR represses expression of the acrAB multidrug efflux genes in Escherichia coli. J Bacteriol. 2008;190(18):6276-9. https://doi.org/10.1128/JB.00190-08 PMID: 18567659

22. Lv F, Cai J, He Q, Wang W, Luo Y, Wang X, et al. Overexpression of Efflux Pumps Mediate Pan Resistance of Klebsiella pneumoniae Sequence Type 11. Microb Drug Resist. 2021;mdr.2020.0395. https://doi.org/10.1089/mdr.2020.0395 PMID: 33835874

23. Telke AA, Olaitan AO, Morand S, Rolain JM. soxRS induces colistin hetero-resistance in Enterobacter asburiae and Enterobacter cloacae by regulating the acrAB-tolC efflux pump. J Antimicrob Chemother. 2017;72(10):2715-21. https://doi. org/10.1093/jac/dkx215 PMID: 29091215

24. Capone A, Giannella M, Fortini D, Giordano A, Meledandri $M$, Ballardini $M$, et al. High rate of colistin resistance among patients with carbapenem-resistant Klebsiella pneumoniae infection accounts for an excess of mortality. Clin Microbiol Infect. 2013;19(1):E23-30. https://doi.org/10.1111/14690691.12070 PMID: 23137235

25. Papadimitriou-Olivgeris M, Christofidou M, Fligou F, Bartzavali $\mathrm{C}$, Vrettos T, Filos KS, et al. The role of colonization pressure in the dissemination of colistin or tigecycline resistant KPCproducing Klebsiella pneumoniae in critically ill patients. Infection. 2014;42(5):883-90. https://doi.org/10.1007/s15010014-0653-x PMID: 25008195

26. Olaitan AO, Diene SM, Kempf M, Berrazeg M, Bakour S, Gupta SK, et al. Worldwide emergence of colistin resistance in Klebsiella pneumoniae from healthy humans and patients in Lao PDR, Thailand, Israel, Nigeria and France owing to inactivation of the $\mathrm{PhoP} / \mathrm{PhoQ}$ regulator mgrB: an epidemiological and molecular study. Int J Antimicrob Agents. 2014;44(6):500-7. https://doi.org/10.1016/j. ijantimicag.2014.07.020 PMID: 25264127 
27. Gona F, Rossi M, Chatenoud L, Itri T, Castelli D, Cavallero A, et al. Impact of plasmids: analysis of the spread of carbapenemresistant Klebsiella pneumoniae epidemic clones in an Italian multi-centre study (Oral communication). 29th ECCMID congress; Amsterdam, Netherlands, 13-16 April 2019.

28. Errico G, Di Pilato V, Monaco M, Giani T, Del Grosso $M$, Antonelli $A$, et al. The changing epidemiology of carbapenemase-producing Klebsiella pneumoniae from invasive infections in Italy: emergence of new high-risk clones (Oral communication). 29th ECCMID congress; Amsterdam, Netherlands, 13-16 April 2019.

29. Shamina O, Kryzhanovskaya O, Alyabyeva N, Lazareva A, Mayanskiy N. Mechanisms of colistin resistance in carbapenem-resistant Klebsiella pneumoniae (Poster). 29th ECCMID congress; Amsterdam, Netherlands, 13-16 April 2019.

30. Tsui KM, Sundararaju S, Al Mana H, Hasan MR, Roscoe D, Thomas E, et al. Genomic characterization of extendedspectrum beta-lactamase in Escherichia coli and Klebsiella pneumoniae in the pediatric population in Qatar (Poster). 29th ECCMID congress; Amsterdam, Netherlands, 13-16 April 2019.

31. Lo S, Goldstein V, Rondinaud E, Ruppé E, Lolom I, Petitjean $M$, et al. Systematic genomic analysis of NDM-producing Enterobacteriaceae during an outbreak in a French university hospital (Poster). 29th ECCMID congress; Amsterdam, Netherlands, 13-16 April 2019.

32. Hoog KJ, Lowe M, Said M, Rule R, Ehlers MM, Pitout JDD, et al. Dissemination of high-risk clonal group 307 amongst extended-spectrum beta-lactamase producing Klebsiella pneumoniae isolates in neonatal and paediatric wards in Tshwane, South Africa (Oral communication). 29th ECCMID congress; Amsterdam, Netherlands, 13-16 April 2019.

33. Lopez JS, Massone CA, Moreno Nunez P, Lopez Fresnena N, Morosini Reilly MI, Canton R, et al. Dissemination of KPC-2-producing Klebsiella pneumoniae ST307 in a tertiary hospital in Madrid (Spain) associated with the emergence of ceftazidime-avibactam resistance (Oral communication). 29th ECCMID congress; Amsterdam, Netherlands, 13-16 April 2019.

34. Jati AP, Perez-Vazquez M, Schouls L, Oteo J, Sola-Campoy PJ, Bosch T, et al. Virulence profiling of OXA-48-producing Klebsiella pneumoniae from Spain and the Netherlands using whole-genome sequencing (Poster). 29th ECCMID congress; Amsterdam, Netherlands, 13-16 April 2019.

35. Chen C, Wu T, Burgess D, Lee G. The resistome and molecular basis of colistin resistance in carbapenem-resistant Klebsiella pneumoniae strains at a large academic medical centre (Oral communication). 29th ECCMID congress; Amsterdam, Netherlands, 13-16 April 2019.

36. Baron SA, Cassir N, Mékidèche T, Mlaga KD, Brouqui P, Rolain $J-M$. Successful treatment and digestive decolonisation of a patient with osteitis caused by a carbapenemase-producing Klebsiella pneumoniae isolate harbouring both NDM-1 and OXA-48 enzymes. J Glob Antimicrob Resist. 2019;18:225-9. https://doi.org/10.1016/j.jgar.2019.06.001 PMID: 31201994

37. Aires CAM, Pereira PS, Asensi MD, Carvalho-Assef APD. mgrB Mutations Mediating Polymyxin B Resistance in Klebsiella pneumoniae Isolates from Rectal Surveillance Swabs in Brazil. Antimicrob Agents Chemother. 2016;60(11):6969-72. https:// doi.org/10.1128/AAC.01456-16 PMID: 27620478

38. Cheng Y-H, Lin T-L, Pan Y-J, Wang Y-P, Lin Y-T, Wang J-T. Colistin resistance mechanisms in Klebsiella pneumoniae strains from Taiwan. Antimicrob Agents Chemother. 2015;59(5):2909-13. https://doi.org/10.1128/AAC.04763-14 PMID: 25691646

\section{License, supplementary material and copyright}

This is an open-access article distributed under the terms of the Creative Commons Attribution (CC BY 4.0) Licence. You may share and adapt the material, but must give appropriate credit to the source, provide a link to the licence and indicate if changes were made.

Any supplementary material referenced in the article can be found in the online version.

This article is copyright of the authors or their affiliated institutions, 2021. 